\title{
L'autoritarisme du verdissement hydroélectrique au Panama : les paradoxes dérégulateurs de l'action climatique vus depuis le Sud
}

\section{The authoritarianism of hydroelectric power greening in Panama: the deregulating paradoxes of climate action seen from the South}

\section{Marie-Esther Lacuisse}

Numéro 82, 2019

Engagements et action publique face à la crise climatique

URI : https://id.erudit.org/iderudit/1061877ar

DOI : https://doi.org/10.7202/1061877ar

Aller au sommaire du numéro

\section{Éditeur(s)}

Lien social et Politiques

ISSN

1703-9665 (numérique)

Découvrir la revue

Citer cet article

Lacuisse, M.-E. (2019). L’autoritarisme du verdissement hydroélectrique au Panama : les paradoxes dérégulateurs de l'action climatique vus depuis le Sud. Lien social et Politiques, (82), 78-95. https://doi.org/10.7202/1061877ar
Résumé de l'article

Cet article interroge les effets dérégulateurs du Mécanisme de développement propre (MDP). Créé en 2001 par les Nations Unies, ce mécanisme incite les pays industrialisés soumis à des objectifs de réduction d'émissions de gaz à effet de serre dans le cadre du protocole de Kyoto à investir dans les énergies non polluantes au Sud en échange de crédits-carbone. L'objectif de ce "verdissement énergétique " est de favoriser la réduction du réchauffement climatique et de stimuler un développement durable au Sud, notion répondant à des buts de justice environnementale et de protection des ressources. À partir du cas du développement de l'énergie hydroélectrique au Panama, cet article montre à l'encontre de cette justification que les critères de la certification propre soutiennent des pratiques économiques ultralibérales qui s'opposent aux principes du développement durable et qui sont à même de conforter des politiques de développement autoritaire. Il donne à voir de ce fait que la valorisation de l'enjeu climatique permet de réaffirmer les principes de la théorie de la modernisation dans le champ du développement en affaiblissant des initiatives durables antérieures. Dans ce cadre, cet article ouvre une réflexion sur les enjeux économiques et politiques de cet effet de régression au regard de la problématique de la souveraineté gouvernementale au Sud. Il développe une sociologie compréhensive du MDP à partir de conflits locaux autour des barrages au Panama. Il valorise une approche analytique depuis la réception, ce qui contribue à approfondir la théorie critique sur ce mécanisme, jusqu'ici principalement étudié depuis ses caractéristiques techniques. 


\title{
L'autoritarisme du verdissement hydroélectrique au Panama : les paradoxes dérégulateurs de l'action climatique vus depuis le Sud
}

\author{
MARIE-ESTHER LACUISSE \\ Docteure en science politique associée \\ au CERIC - UMR DICE 7318 - CNRS / Aix-Marseille Université
}

\section{Introduction}

Dans le cadre de la politique internationale de lutte contre le réchauffement climatique, un instrument a été mis en place par les Nations Unies pour soutenir le verdissement des énergies dans les pays en voie de développement et dans les pays émergents. II s'agit du Mécanisme de développement propre (MDP), élaboré en 2001 dans le cadre du protocole de Kyoto (1997-2020) et qui devrait être maintenu pour soutenir les accords de Paris ${ }^{2}$. Ce mécanisme incite les entités publiques et privées des pays industrialisés soumis à des réductions d'émissions de gaz à effet de serre à investir dans le développement d'énergies non polluantes au Sud en échange de crédits-carbone que les investisseurs peuvent garder ou revendre. Les pays receveurs ont ainsi accès à une énergie verte sans assumer de coût d'investissement.

Selon les Nations Unies, ce mécanisme a pour but de réduire l'émission de carbone à l'échelle mondiale, tout en stimulant un développement durable au $\mathrm{Sud}^{3}$ : un développement à dimension sociale et écologique visant à protéger les générations présentes et futures de la surconsommation des ressources, selon la définition de la notion par l'organisation internationale en 1987 (Rist, 2007; Pestre, 2011). À partir du cas du développement de l'énergie hydroélectrique au Panama, nous verrons à l'encontre de cette justification que le MDP soutient des pratiques économiques ultralibérales qui s'opposent aux principes du développement durable, et qui confortent, de plus, des

(CIien social et Politicutes no $82-2019$

ENGAGEMENTS ET ACTION PUBLIQUE FACE À LA CRISE CLIMATIQUE 
politiques de développement autoritaire. Cet article montre, dans ce sens, que la certification propre ne considère pas les impacts socio-écologiques des projets soumis à l'évaluation, mais uniquement leur contribution additionnelle à la réduction des émissions de gaz à effet de serre et que, de ce fait, le mécanisme permet de libérer les investissements des contraintes de mesures durables élaborées au cours des années 1990 dans le but, entre autres, de réduire les coûts socio-environnementaux des investissements de l'aide au développement au Sud. II donne à voir dans ce cadre que la certification propre $n$ 'intègre pas dans ses critères d'évaluation le contenu des normes socio-environnementales des banques multilatérales de développement (BMD) ni les recommandations de la Commission mondiale des barrages $(\mathrm{CMB})$, qui, bien que relevant de la «soft law», un droit mou non contraignant juridiquement, ne sont pas sans créer des contraintes sur la mise en œuvre des projets.

Cet article propose ainsi une lecture critique de l'impact du MDP à l'échelle d'un pays receveur, soit de ses conséquences dans les pays du Sud. II montre dans ce cadre comment le MDP, un mécanisme présenté comme une innovation relevant d'une extension du droit de la terre à celui de l'atmosphère (Godard, 2015), est également empreint d'un air de régression.

Il avance le fait que la promotion de l'enjeu climatique participe de manière paradoxale à réaffirmer les principes économiques du développement de la théorie de la modernisation (Lipset, 1959) contre lesquels les premières initiatives durables se sont forgées. En d'autres termes, cet article souligne les effets de dérégulation de la certification propre dans le champ du développement autant que dans celui de l'environnement qui lui est lié, et contribue, dans cette perspective, à approfondir et à renouveler la théorie critique sur le MDP qui a principalement été développée, jusqu'à présent, depuis les caractéristiques techniques de l'instrument. Alors que des travaux ont mis en avant les travers d'un mécanisme de marché participant à la néolibéralisation de la régulation environnementale (Harrison et Milker, 2014) et à la modernisation écologique du capitalisme (Aykut et Dahan, 2015) en tant qu'«économie de la promesse » qui profite à l'ouverture de nouveaux marchés (Foyer, Viard et Boisvert, 2017), d'autres travaux ont souligné les insuffisances des critères de la certification propre pour soutenir une dynamique de transition (Newell et al., 2009) et un développement durable au Sud (Tsaymen, 2013b). 
Cet article s'inscrit dans une démarche de sociologie compréhensive de I'action publique qui consiste à saisir la rationalité de l'action non seulement par le bas (Le Galès et Lascoumes, 2007; Snyder, 2001), mais aussi depuis sa réception (Révillard, 2018), ainsi que depuis ses effets. II s'appuie sur une enquête réalisée auprès de contestataires de barrages au Panama dans le cadre d'un projet de recherche sur les mécanismes de plainte des BMD. Ces mécanismes permettent aux populations locales, lorsqu'elles considèrent subir les dommages d'un projet financé par les banques, de réclamer à ces dernières l'application de leurs normes socio-environnementales. Les plaignants peuvent espérer en retour des mesures correctives, si un rapport de non-conformité est produit ${ }^{5}$. Dans le cadre de cette recherche, deux plaintes émises depuis le Panama ont été étudiées; et les entretiens effectués avec douze plaignants, ainsi que la lecture des documents liés aux requêtes (plaintes et rapport de conformité) ont conduit, d'une part, à prendre la mesure du développement autoritaire de lénergie hydroélectrique au Panama et, d'autre part, à considérer l'implication des critères de la certification propre dans ce processus.

Compte tenu de l'approche empirique des effets dérégulateurs du MDP proposée dans ce texte, approche qui s'appuie sur des conflits locaux, une première partie sera consacrée à un état des lieux et des acteurs du développement autoritaire de l'énergie hydroélectrique au Panama à partir de nos deux études de cas. Nous développerons ensuite d'un point de vue théorique la manière dont la certification propre agit, dans le champ de la régulation environnementale, comme un levier de dérégulation en marquant un retour au laissez-faire, dont nous discuterons des enjeux politiques dans une dernière partie. Nous verrons dans ce cadre que la certification propre permet de faciliter les investissements, mais aussi de libérer les gouvernements des pays du Sud de pressions exogènes dans une perspective de rééquilibrage de l'exercice du néolibéralisme entre Nord et Sud. Enfin, la conclusion débattra du caractère extrême de notre étude de cas - l'énergie hydroélectrique au Panama - en ce qui concerne les impacts négatifs du MDP au Sud. 


\section{1. État des lieux et des acteurs du développement autoritaire de l'hydroélectrique au Panama}

Depuis le milieu des années 2000, des entreprises européennes en participation, mais aussi nord-américaines et latino-américaines, ainsi que les banques de développement, multilatérales autant que d'investissements, ont investi dans le secteur de l'énergie hydroélectrique au Panama, un pays riche en eau avec 119 milliards de mètres cubes disponibles et environ 500 cours d'eau', dans le cadre de la politique internationale du développement propre et d'un plan de développement régional. II s'agit du Plan Puebla Panama, élaboré à l'initiative du Mexique en 2001 (Collombon, 2017), puis renommé Projet mésoaméricain en 2008. L'un de ses composants majeurs est l'interconnexion électrique non polluante de la région de l'Amérique centrale afin de soutenir le développement économique durable de pays pauvres. Si ce verdissement énergétique a pu contribuer à réduire les émissions de carbone, il s'est fait de manière autoritaire et ultralibérale, en allant contre la protection des ressources naturelles et des populations locales, et cela, en accord avec les critères de certification du MDP. C'est ce que nous allons voir avec l'enquête menée auprès des usagers des mécanismes de plaintes dans les provinces de Chiriqui et de Bocas del Toro.

\subsection{La noyade de Chiriqui sous les barrages du verdissement climatique}

L'enquête de terrain au Panama a tout d'abord débuté dans le nord de la région de Chiriqui, frontalière du Costa Rica, où des agriculteurs et des militants écologistes locaux avaient déposé une requête auprès des mécanismes de plainte de la Banque interaméricaine de développement (BID) et de la Société financière internationale (SFI), banque responsable des investissements dans le privé du Groupe de la Banque mondiale, à propos de la construction de deux barrages de 33 et 54 mégawatts sur la rivière Chiriqui Viejo. Par cette requête déposée en 2010, les plaignants cherchaient à dénoncer non seulement le projet surdimensionné des barrages Pando et Montelirio, développé par une entreprise en participation panaméenne-espagnole, mais aussi l'accumulation sur le cours d'eau de projets hydroélectriques sans régulation du secteur ni consultation de la population locale ${ }^{7}$. Comme a pu le dire un plaignant : «lls nous ont pris de surprise avec les barrages ${ }^{8} \gg$. 
En effet, lorsque la plainte a été déposée, la rivière Chiriqui Viejo, d'une longueur de $161 \mathrm{~km}$, comptait déjà neuf projets hydroélectriques, dont sept étaient en activité. Selon un des six plaignants interrogés, l'objectif de la plainte était : «d'avertir la BID et la SFI que la politique énergétique dans notre pays était nouvelle et très fragile. Nous voulions les inciter à faire attention à cela avant d'approuver le projet. Ce n'est pas tant l'entreprise privée que nous visions dans nos plaintes que l'incapacité des autorités publiques à gérer de tels projets ${ }^{9} \gg$. Alors que la BID et la SFI disposent de normes visant à réduire les impacts socio-environnementaux des projets en exigeant notamment des études d'impact cumulatif permettant d'évaluer l'incidence d'un projet au regard des projets déjà existants (BID, 2006; SFI, 2007), le Panama ne disposait pas d'instrument à même de réguler la construction des barrages ni I'usage de l'eau. La seule loi existante était alors, et reste encore, la résolution législative sur la gestion du flux écologique des cours d'eau, qui ne prévoit de réserver que $10 \%$ de l'eau aux écosystèmes. Les $90 \%$ restants sont d'usage libre sans mesure de partage de la ressource entre les activités productives ${ }^{10}$.

Cet appel à la BID et à la SFI pour intervenir dans la régulation du bassin n'a cependant pas eu les effets escomptés, bien que le mécanisme de plainte de la BID ait réalisé un rapport de conformité qui valide les problèmes dénoncés par les plaignants concernant la gestion écologique du bassin"1. Les barrages ont été approuvés et construits. Surtout, les projets hydroélectriques ont continué de se multiplier dans les bassins de la province de Chiriqui sous l'effet des réformes libérales et autoritaires du président de la République Ricardo Martinelli (2009-2014). Tout d'abord, en juillet 2010, le président Martinelli a promulgué un décret prévoyant la simplification des études d'impact environnemental sur les projets d'investissement dans le cadre d'une loi restreignant le droit à la manifestation et à la grève, surnommée «Loi Chorizo» au Panama12. Puis, en 2013, il a soutenu un décret-loi facilitant les expropriations pour accélérer l'implantation de projets hydroélectriques. Le texte autorisait les entreprises à exproprier de force les propriétaires des terres le long des rivages, si elles n'étaient pas parvenues, dans un délai d'un mois, à négocier avec eux ${ }^{13}$.

De fait, lorsque l'enquête a été réalisée en janvier 2015, le nombre de projets hydroélectriques sur la Chiriqui Viejo n'était plus de onze, mais de vingt-six, et la province de Chiriqui comptait plus de 73 concessions hydroélectriques ${ }^{14}$, ce qui représente les trois quarts des concessions octroyées au secteur au Panama. Face à cette dérégulation autoritaire, qui peut être comprise comme 
l'expression d'un ultralibéralisme donnant les pleins pouvoirs aux investisseurs, ce n'étaient plus seulement les habitants du bassin de Chiriqui Viejo qui étaient mobilisés contre les barrages, mais la population de plusieurs bassins, inquiète de la manière dont un nouveau secteur d'activité s'accaparait l'eau des rivières et menaçait du même coup l'agriculture d'une région qui fournit le Panama en fruits et légumes, les écosystèmes, ainsi que la vie des habitants. Par exemple, lors de la réalisation du second terrain d'enquête en juin 2016, une centrale hydroélectrique était en train d'être construite sur le point d'eau potable d'un village au bord de la rivière Macho del Monte. II s'agit de la centrale La Cuchilla de neuf mégawatts de l'entreprise panaméenne Hidropiedra S.A, financée par des investissements d'une entreprise espagnole.

La majorité des projets hydroélectriques développés dans la province de Chiriqui n'ont pas été certifiés «propres» par le MDP. Ils n'ont même pas forcément été construits pour obtenir cette certification. Sur les 23 projets certifiés «propres» au Panama, 18 sont des projets hydroélectriques et une dizaine ont été exécutés dans la province de Chiriqui ${ }^{15}$. Or, l'enquête a révélé le fait que le développement autoritaire et ultralibéral de l'énergie hydroélectrique à Chiriqui était lié à la politique du «développement propre», qui accorde la certification à des projets en ne prenant en considération que leur contribution carbone, soit «l'additionnalité » des projets à la réduction des émissions de gaz à effet de serre. Ce critère climatique du MDP incite les entreprises à construire des ouvrages de grandes tailles, car seuls ces derniers peuvent contribuer à la réduction de carbone et obtenir, par conséquent, la certification propre. C'est le cas des barrages Pando et Montelirio, développés en enfilade sur la rivière Chiriqui Viejo, et qui ont été certifiés en 2017. C'est aussi le cas du barrage Bajo Frio de 57 mégawatts, développé en aval de la même rivière par une entreprise en participation panaméenne norvégienne et avec l'appui de Proparco, la banque d'investissement de l'Agence française de développement. Le MDP peut aussi encourager les gouvernements à mener des réformes ultralibérales, la certification n'étant pas assujettie à des études d'impact environnemental consistant à évaluer les conséquences écologiques et sociales des projets, comme peuvent l'exiger les normes des BMD. De fait, le «développement propre» déconsidère les conditions d'exécution des projets. L'un des six plaignants interrogés à Chiriqui a d'ailleurs questionné l'impact négatif de la valorisation du climat en ces termes : «Encore une fois, on se moque de nous. On prétend que ces hydro servent la planète en réduisant l'émission de gaz à effet de serre. Mais, pendant ce temps, que devient notre bassin ${ }^{16}$ ? 》 
Cette implication du «développement propre» dans le verdissement énergétique autoritariste au Panama s'est ensuite confirmée dans la province voisine de Bocas del Toro, où l'enquête sur les mécanismes de plainte a conduit à s'intéresser à la construction de grands barrages dans l'espace de vie de communautés indiennes qui avaient déjà fait l'objet d'une mobilisation contre leur certification et contre les critères de la certification propre.

\subsection{Les Indiens de Bocas à l'épreuve de grands barrages «propres»}

L'objet de l'enquête à Bocas del Toro concernait une requête déposée auprès du mécanisme de plainte de la Banque mondiale (BM) à propos du détournement d'un projet de réforme agraire au profit d'entreprises privées. Parmi leurs doléances, les plaignants contestaient la construction de grands barrages dans l'espace de vie de communautés indiennes qui auraient normalement dû être protégées de toute activité commerciale et extractive avec le projet de réforme agraire financé par la BM. Ce projet prévoyait en effet, conformément à la politique de protection des peuples indigènes de la $\mathrm{BM}^{17}$, de préserver les espaces de vie des communautés indiennes vivant hors des territoires communautaires (nommés «comarcas 》 au Panama ${ }^{18}$ ) de toute concession. Or, en 2008, après l'approbation du financement du projet par la BM, le président Martin Torrijos (2004-2009) a modifié le texte de loi en restreignant l'usage communautaire au profit des investissements privés. Des concessions ont dès lors pu être accordées dans les espaces de vie de communautés indiennes, et certaines communautés ont été déplacées ou divisées par la construction de grands barrages sur «leur terre». Les principaux barrages contestés étaient le projet hydroélectrique Bonyic de 33 mégawatts de l'entreprise publique colombienne Empresa Medellin et le mégabarrage Chan 75 d'une capacité de 222 mégawatts développé par la filiale panaméenne de l'entreprise nord-américaine AES. Le premier avait été construit au milieu des terres de la communauté Naso. Le second avait été aménagé sur la rivière Changuinola dans la zone d'amortissement du parc Amistad, classé par l'UNESCO, et il avait provoqué le déplacement violent de plus de 1000 membres de la communauté Ngöble-Buglé. Face aux conséquences de la construction de ce barrage, des membres de la communauté Ngöble-Buglé, dont un des plaignants interrogés, avaient déposé un recours auprès de la Commission interaméricaine des droits humains, car le projet ne respectait pas les droits internationaux des peuples indigènes dans un pays qui, de surcroît, venait de ratifier la Déclaration des Nations Unies sur les droits des peuples autochtones. 
Par ailleurs, deux des cinq plaignants interrogés ont mentionné le fait que ces deux projets avaient été inscrits sur la liste des projets «propres » du Panama, mais qu'une mobilisation transnationale contre leur certification avait contribué à ce qu'ils ne soient finalement pas certifiés ${ }^{19}$. En 2008, un an avant le dépôt de la plainte à la BM, des organisations non gouvernementales nord-américaines et panaméennes avaient en effet envoyé des lettres aux membres de l'organisme certificateur afin de dénoncer la faible contribution des projets au développement durable et le «greenwash additionnality» que représenterait leur certification ${ }^{20}$. Par cette expression, qui joue avec le critère $d^{\prime}$ «additionnalité » des projets de réduction des émissions de gaz à effet de serre, ces ONG dénoncent une certification qui soutiendrait, si elle était accordée, l'écoblanchiment de projets servant avant tout des intérêts commerciaux et économiques dans un pays déjà autosuffisant en ressource hydroélectrique. Une de ces lettres mentionne en outre que le MDP lui-même ne respecte pas les principes du développement durable en n'appliquant pas les recommandations de la Commission mondiale des barrages. En 2000, cette commission, sur laquelle nous allons revenir par la suite, a en effet formulé des recommandations à destination des entreprises privées et des États dans le but de réduire les dégâts socio-environnementaux de la construction des grands barrages au Sud. À ce sujet, un plaignant de Bocas a d'ailleurs qualifié le MDP «d'écran de fumée environnemental ${ }^{21}$ ». II a précisé, pour justifier son propos, qu'à deux reprises des BMD avaient fait marche arrière sur le financement de barrages inscrits sur la liste des projets «propres » du Panama à cause de leur impact socio-écologique et, pourrait-on ajouter, des retombées médiatiques négatives du financement de tels projets. II a tout d'abord mentionné le cas de la BID, qui devait financer le projet Bonyic avant de renoncer. Puis il a évoqué le cas de la Banque européenne d'investissement, qui s'était retirée du financement du grand barrage Barro Blanco, construit sur la rivière Tabasará dans la comarca Ngöble-Buglé. Ce grand barrage de 29 mégawatts et disposant d'un réservoir de $2,5 \mathrm{~km}^{2}$ relevait de la catégorie des ouvrages publics. Construit par une entreprise hondurienne et, finalement, financé par des banques d'investissement européennes ${ }^{22}$, il a été certifié «propre» par le MDP en 2011. 


\section{La certification propre et la réaffirmation du «laissez-faire» contre les critères de la durabilité}

Il est ainsi ressorti de cette enquête que le mécanisme du «développement propre» permet la mise en œuvre de projets qui ne respectent pas les principes du développement durable ni même les droits internationaux des peuples autochtones. C'est ce que deux chercheurs ayant travaillé sur les cas de Chan 75 et Bonyic ont nommé «l'autoritarisme vert» (Finley-Brook et Curtis, 2013), en soulignant les insuffisances d'un tel mécanisme. Notre approche du MDP, développée à partir des conflits autour des barrages, nous permet toutefois d'affirmer que les impacts négatifs du MDP ne répondent pas qu'à des externalités négatives, dans la mesure où la certification propre $n^{\prime}$ intègre pas des standards de production durables préexistants, telles les normes des BMD et les recommandations de la Commission mondiale sur les barrages.

Par cette observation, notre but n'est pas d'opposer des approches du développement de différentes organisations. Nous avons d'ailleurs vu qu'en pratique les BMD ne s'en tiennent pas toujours à leurs principes d'action ou qu'elles ont, tout du moins, des comportements contradictoires, telle la BID qui ne finance pas un grand barrage dans l'espace de vie d'une communauté indienne, mais soutient le Plan Puebla Panama pourtant à l'origine de la prolifération des projets hydroélectriques au Panama. Notre propos vise plutôt à souligner le fait que la certification propre contribue à réaffirmer les principes économiques du développement contre lesquels ont été élaborées les initiatives durables des années 1990, dans le but de réguler les coûts socioenvironnementaux des investissements au Sud, et tout particulièrement ceux dans le secteur de l'énergie hydroélectrique.

Les normes des BMD et les recommandations de la Commission mondiale sur les barrages qui en découlent sont en effet, en partie, la résultante d'une mobilisation contre les dommages occasionnés par l'aide au développement au Sud. Cette mobilisation a débuté au milieu des années 1980 en prenant pour cible les investissements de la BM. Des ONG environnementalistes nord-américaines, dont International Rivers et Center for International Environnemental Law, désormais investies dans la contestation de la certification propre ${ }^{23}$, ont alors fait des dommages provoqués par les projets de développement financés par la BM l'argument majeur de leur plaidoyer en faveur d'une nouvelle approche du développement qui sorte des principes 
économiques de la théorie de la modernisation (Lipset, 1959; Rist, 2007). Ces ONG se sont mobilisées pour l'introduction de critères de durabilité qui visent la protection des ressources naturelles et les droits des populations les plus vulnérables, recoupant les enjeux de la justice environnementale (Larrère, 2009). Pour ce faire, elles ont organisé une mobilisation transnationale avec des relais militants dans les zones de mise en œuvre des projets et des appuis dans les arènes politiques des États bailleurs aux États-Unis (Keck et Sikkink, 1998), mais aussi en Europe (Van Putten 2008); et elles ont mobilisé pour leur première campagne les dommages du mégaprojet hydroélectrique Sardar Sadovar en Inde. La BM devait financer la construction d'un réseau de canaux de plus de $440 \mathrm{~km}$ reliant plusieurs barrages construits autour d'un réservoir de plus de $210 \mathrm{~km}$ sans qu'un plan de relocalisation ait été prévu pour les centaines de milliers de personnes affectées (Clark, 2003).

Cette première campagne, ainsi qu'une suivante sur un projet forestier au Brésil, a soutenu la publication d'une norme «Évaluation environnementale» à la BM en 1989. Cette dernière exige des études d'impact environnemental sur les projets soumis à financement, incluant l'analyse des aspects purement environnementaux comme la pollution et les écosystèmes, mais aussi sociaux comme les conditions de subsistance des populations locales. Ces campagnes ont aussi permis d'améliorer la directive opérationnelle «Déplacements involontaires des populations » de la BM (Fox et Brown, 1998) en renforçant les exigences en matière de plan de relocalisation, notamment en ce qui concerne la préservation des conditions de vie des déplacés. Ces normes, dont les qualifications ont évolué au fil du temps (directive opérationnelle, politique opérationnelle et, désormais, normes environnementales et sociales), adressent des recommandations aux agents de la banque responsables de la préparation des projets et relèvent d'un droit non contraignant juridiquement.

Ensuite, durant les années 1990, la présidence du Groupe de la Banque mondiale a continué d'élever ses exigences en matière de développement durable, dans un contexte de perte de légitimité de l'action de l'institution financière internationale et sous l'effet des plaintes reçues par le Panel d'inspection de la BM, premier mécanisme de plainte à avoir été créé, en 1993, dans le prolongement de la mobilisation pour les normes (Hunter, 2008). L'application des normes de la BM a alors été étendue à la SFI, donc aux investissements du Groupe de la Banque mondiale dans le secteur privé, et des mesures spécifiques ont été prises pour réduire les dommages provoqués 
par la construction des barrages au Sud. En 1996, la BM a notamment publié une directive opérationnelle nommée «sûreté des barrages», qui définit des standards techniques pour éviter les inondations ${ }^{24}$. L'année d'après, le président du Groupe de la Banque mondiale, James D. Wolfensohn (19952005), a alors œuvré avec l'Union internationale pour la conservation de la nature à l'ouverture d'une Commission mondiale sur les barrages.

Cette commission, composée d'acteurs politiques et de militants en provenance du Nord et du Sud, a abouti en 2000 à la publication d'un rapport de recommandations nommé «Barrages et développement : un nouveau cadre pour la prise de décision», destiné aux entreprises des pays industrialisés et des États au Sud (World Commission on Dams, 2000). Ce rapport met notamment en garde les entreprises contre la construction de grands barrages surdimensionnés sujets à des inondations et à l'origine du déplacement de personnes. II recommande également de considérer les effets cumulatifs, c'est-à-dire de prendre en compte les autres centrales déjà en construction et en projet sur une rivière, à la manière de ce que font la BID et la SFI. En bref, ce rapport recommande des mesures de développement durable similaires à celles des BMD, mesures que le MDP, créé une année plus tard lors des accords de Marrakech sur le climat en 2001 (Maljean-Dubois, 2005; TsaymenDemaze, 2013a), n'applique pas, alors même que le mécanisme a vocation à soutenir des projets hydroélectriques. Sur les 7805 projets «propres» validés par le MDP depuis sa création, à peu près 1708 d'entre eux relèvent du secteur de l'énergie hydroélectrique ${ }^{25}$.

Ainsi, et alors même que le MDP encourage l'innovation à l'aide d'actions climatiques censées soutenir une transition écologique, la certification propre opère un effet de dérégulation favorable aux investissements en les libérant de normes et de recommandations relevant certes de la soft law, mais pas pour le moins contraignantes. En effet, en n'attachant pas d'importance aux standards de la durabilité, le MDP libère les investissements de la charge de réaliser des études d'impact environnemental qui, d'une part, complexifient et retardent les projets (plus qu'elles ne les menacent) et, d'autre part, entraînent des coûts économiques additionnels liés à l'aménagement d'un plan de relocalisation ou encore à la recherche d'alternatives technologiques pour réduire les dommages occasionnés par les projets. Le MDP n'exige d'ailleurs aucun critère en matière de transfert de technologie au Sud, problème qu'ont pu pointer des chercheurs qui ont analysé cet instrument d'un point de vue technique (Tsaymen-Demaze, 2013b). 


\section{Entre facilitation des investissements et enjeu de souveraineté néolibérale au Sud}

Vu depuis un pays receveur, le MDP se caractérise ainsi moins par son recours à une «économie de la promesse» porteuse de modernisation «écologique» du capitalisme (Aykut et Dahan, 2015) en ouvrant des nouveaux marchés d'expertise, que par la consécration d'un ultralibéralisme vert voué à dégager les investissements de toute contrainte.

Cette réaffirmation d'un certain laissez-faire ne doit cependant pas être réduite à des enjeux économiques liés à l'intérêt des entreprises des pays industrialisés. Bien au contraire, peut-on ajouter, si l'on considère que les contraintes technologiques sont favorables aux entreprises des pays industrialisés, comme le suggèrent des travaux réalisés sur les initiatives de régulation environnementale des États-Unis dans les années 1970 (De Sombre, 2000). À travers nos études de cas, nous avons d'ailleurs vu que les barrages du «développement propre» sont souvent construits par des entreprises des pays du Sud avec le soutien financier d'entités européennes : entreprises et banques d'investissement. Les critères du «développement propre» ouvrent ainsi potentiellement de nouveaux marchés pour les entreprises des pays du Sud.

L'effet dérégulateur du MDP est également à envisager d'un point de vue politique. II permet aux gouvernements des pays du Sud de se dégager des obligations venant d'instruments de soft law, empiétant sur leur souveraineté et allant contre les principes néolibéraux qui leur ont été imposés à partir des années 1980. En effet, à partir des années 1980, il a été demandé aux gouvernements des pays du Sud et d'Amérique latine, en premier lieu, d'engager une réforme néolibérale de l'État consistant à réduire son interventionnisme aux profits des investissements privés dans le cadre de l'application des principes du Consensus de Washington. II a notamment été exigé des gouvernements qu'ils diminuent les dépenses publiques et qu'ils rationalisent les administrations. Parallèlement, les normes de développement durable déployées ont, quant à elles, exigé des États qu'ils mettent en place des politiques et développent des compétences en matière environnementale. Par exemple, une étude d'impact cumulatif nécessite des savoirs et des politiques publiques en gestion des ressources qui ne relèvent pas seulement des compétences de l'entreprise, mais aussi des autorités du pays où est mis en œuvre le projet. $\mathrm{Si}$, depuis une perspective globale, les normes du développement durable 
des années 1990 soutiennent une régulation des effets négatifs du libéralisme s'inscrivant dans un projet de société plus protecteur à l'égard des individus et de la nature, elles sont également à envisager, à l'échelle des gouvernements récipiendaires de l'aide et des investissements, comme des instruments de contrôle qui s'opposent à la logique des réformes néolibérales imposées.

En partant de ce point de vue, il devient ainsi pertinent d'envisager le MDP comme un instrument participant à un rééquilibrage de l'exercice du néolibéralisme entre Nord et Sud, et à une affirmation de la souveraineté gouvernementale au Sud. En ne prenant en compte que la contribution du projet à la réduction de carbone, le MDP permet en effet aux États du Sud de se libérer de pressions et de contraintes exogènes. II est d'ailleurs intéressant de noter que, si certains travaux attribuent la genèse du mécanisme aux États-Unis (Aykut et Dahan, 2015) avant que ce pays ne quitte les accords sur le climat en 2001, d'autres travaux attribuent cette initiative à un pays du Sud: le Brésil (Tsayem, 2010). Cette idée nous permet par ailleurs de souligner un des points les plus minimisés du fonctionnement du MDP, surtout dans les travaux qui abordent cet instrument sous l'angle d'un mécanisme de marché : le règlement du MDP précise que la mise en œuvre d'un projet ne peut se faire que selon la législation en vigueur dans l'État receveur, ce qui valorise l'idée d'un laissez-faire politique.

Dans cette perspective, il faut enfin ajouter que la soumission d'un projet au MDP ne se fait pas sans l'accréditation des États receveurs et que ces derniers ont d'ailleurs le droit de retirer leur accréditation à un projet, qui perdra alors son label «développement propre» et l'entreprise privée ses crédits-carbone. Face à la mobilisation sociale contre le grand barrage Barro Blanco, qui s'est renforcée au moment du second séjour d'enquête, après que des terres ont été inondées avec l'ouverture des vannes du barrage, le président panaméen Juan Carlos Varela (2014-2019) a d'ailleurs retiré l'accréditation «propre» au barrage. En novembre 2016, Barro Blanco est devenu le premier projet «décertifié » par un État. Si cette décertification a empêché l'obtention de crédits-carbone, elle n'a en revanche rien changé à l'échelle locale, le barrage ayant été construit sans que soient pris en considération les critères de la durabilité du projet. 


\section{Conclusion : l'hydroélectrique au Panama, un cas extrême significatif des risques d'autoritarisme}

À partir du cas de l'énergie hydroélectrique au Panama, nous avons ainsi montré qu'en valorisant l'enjeu climatique le MDP discrédite les initiatives durables des années 1990 et permet de cette manière de réaffirmer les principes économiques du développement. En ce sens, cet article rejoint la conclusion des travaux critiques sur la nature marchande du mécanisme - le MDP participant à une modernisation écologique du capitalisme ou à une néolibéralisation de la régulation environnementale -, à la différence près que nous n'avons pas mis l'accent sur la création de nouveaux marchés liés au climat, mais sur le fait que la certification propre agit comme un levier dérégulateur en marquant un retour du laissez-faire favorable aux investissements. Par conséquent, nous pouvons ajouter que le principal obstacle du MDP pour soutenir une transition écologique ne vient pas du fait qu'il est un mécanisme de marché, mais davantage du contenu des critères de la certification. Si le marché participe à la régulation politique (Ansaloni et al., 2017), ce n'est cependant pas le marché en soi qui confère au MDP sa rationalité, mais les règles qui lui sont assujetties et qui, en l'occurrence, peuvent conforter des politiques de développement ultralibérales et autoritaires.

En soulignant les effets de dérégulation du MDP, cet article n'affirme pas pour autant que le «développement propre » entraîne des politiques de verdissement énergétique autoritaires dans tous les États receveurs, tel qu'observé au Panama. Comme nous avons pu le voir, l'absence de considération pour les critères de durabilité répond aussi à des enjeux de gouvernabilité relatifs à la correction des contradictions de l'exercice du néolibéralisme entre Nord et Sud, et à la réaffirmation de la souveraineté des États au Sud par rapport aux pays industrialisés et aux instances internationales. Des gouvernements peuvent tout à fait associer enjeu climatique et développement durable, la mise en œuvre des projets se faisant selon la législation du pays receveur. Notre étude de cas - l'énergie hydroélectrique au Panama - doit donc être envisagée de ce point de vue en tant que cas extrême concernant des impacts négatifs du MDP pour le Sud, mais pas non représentative, pour autant, des risques d'autoritarisme de l'action climatique.

Tout d'abord, notre étude de cas a porté sur une énergie non polluante controversée à cause de ses impacts socio-écologiques au Sud. Nous avons vu que les normes socio-environnementales des BMD trouvent leur origine 
dans une mobilisation transnationale organisée par des ONG, afin de réduire les dommages des grands barrages au Sud. Nous avons également observé que l'action menée par la BM pour réguler les impacts socio-environnementaux de l'aide au développement a conduit à la mise en place de recommandations spéciales pour les barrages. Certaines ONG ont d'ailleurs déclaré que le financement des grands barrages sapait le MDP ${ }^{26}$. En effet, et bien que la certification propre ne soit pas dotée de critères de durabilité, un projet de biomasse proposant des alternatives énergétiques à la production agricole en utilisant des matières d'origine végétale ou bactérienne est moins susceptible d'entraîner des conséquences écologiques et humaines. Enfin, notre étude de cas se situe dans un pays d'Amérique latine, aire culturelle où les processus de modernisation économique ont souvent été liés à des pratiques bureaucratiques autoritaires constitutives des premiers travaux critiques sur la théorie de la modernisation dans les années 1970 (O'Donnell, 1973). Cela dit, il n'en demeure pas moins que des rapports d'ONG tendent à indiquer que le MDP est source de nombreux problèmes locaux à travers le monde (Carbon Market Watch, 2018). En 2018, 61 ONG des pays industrialisés et du Sud ont, pour leur part, envoyé une pétition aux chefs de délégation de la Convention-cadre des Nations Unies sur les changements climatiques, afin que le MDP ne soit pas prolongé après 2020, car les projets développés dans ce cadre ont un trop faible impact sur la réduction de carbone et, surtout, des conséquences humaines trop néfastes, pour qu'ils puissent aller dans le sens des accords de Paris ${ }^{27}$.

\section{Bibliographie}

Ansaloni, Matthieu, Pascale Trompette et Pierre-Paul Zalio. 2017. «Le marché comme forme de régulation politique», Revue française de sociologie, 58, $3: 359-374$.

Aykut, Stephan et Amy Dahan. 2014. Gouverner le climat? Vingt ans de négociations internationales. Paris, Presses de Sciences Po.

Banque interaméricaine de développement. 2006. La Política de Medio Ambiente y Cumplimientos de Salvaguardias. <http://www20.iadb.org/intal/catalogo/PE/2010/07136.pdf>. Page consultée le 15 avril 2015.

Carbon Market Watch. 2018. « The clean development mechanism: local impacts of a global system ». <https://carbonmarketwatch.org/publications/the-clean-developmentmechanism-local-impacts-of-a-global-system>. Page consultée le $1^{\text {er }}$ décembre 2018.

Clark, Dana. 2003. «Understanding the World Bank Inspection Panel», dans Dana Clark, John Fox et K. Treakle (dir.). Demanding Accountability: Civil-Society Claims and the World Bank Inspection Panel. Lanahm, Rownam \& Littlefield Publishers : 1-24. 
Collombon, Maya. 2017. «Écrire l'international. Genèses d'une politique de développement transnational, le Plan Puebla Panama», Critique internationale, 77, 4 : 33-55.

De Sombre, Elizabeth. 2000. Domestics Sources of International Environment Policy: Industry, Environmentalists, and U.S. Power. Massachusetts, MIT Press.

Finley-Brook, Mary et Thomas Curtis. 2013. «Renewable Energy and Human Rights Violations: Illustrative Cases from Indigenous Territories in Panama», dans Karl Zimmerer (dir.). The New Geographies of Energy: Assessment and Analysis of Critical Landscapes. New York, Routledge : 162-171.

Foyer, Jean, Aurore Viard-Crétat et Valérie Boisvert. 2017. « Néolibéraliser sans marchandiser? La bioprospection et les REDD dans l'économie de la promesse», dans Daniel Compagnon et Estienne Rodary (dir.). Les Politiques de la biodiversité. Paris, Presses de Sciences Po : 225-249.

Fox, Jonathan et David Brown (dir.). 1998. The Struggle for Accountability: The World Bank, NGOs, and Grassroots Movements. Massachusetts, MIT Press.

Godard, Olivier. 2015. La justice climatique mondiale. Paris, La Découverte.

Harrison, N.E. et John Mikler. 2014. «An Introduction to Climate Innovation», dans N. E Harrison et John Mikler (dir.). Climate Innovation. Energy, Climate and the Environment Series. Londres, Palgrave Macmillan.

Hunter, David. 2008. « Civil Society Networks and the Development of Environmental Standards at International Financial Institutions », Chicago Journal of International Law, $8,2: 437-477$.

Keck, Margaret et Kathreen Sikkink. 1998. Activists Beyond Borders: Advocacy Networks in International Politics. The World Bank, NGOs, and Grassroots Movements. Ithaca New-York, Cornell University Press.

Lacuisse, Marie-Esther. 2017. «Los mecanismos de rendición de cuentas de los bancos multilaterales de desarrollo como vía de acceso a información medioambiental en América Latina», dans P. Gautreau et N. Monheburrun (dir.). Direito à Informação Ambiental: uma agenda de pesquisa interdisciplinar. Parana, Editora Prismas : 341-372. Version française disponible à la page <https://hal.archives-ouvertes.fr/hal-01314701>.

Larrère, Catherine. 2009. «La justice environnementale», Multitudes, 36 : 156-162.

Lipset, Seymour. 1959. «Some Social Requisites for Democracy: Economic Development and Political Legitimacy », American political science review, 53, 1: 69-105.

Le Galès, Patrick et Pierre Lascoumes. 2007. Sociologie de l'action publique. Paris, Armand Colin.

Maljean-Dubois, Sandrine. 2005. «La mise en route du protocole de Kyoto à la conventioncadre des Nations Unies sur les changements climatiques», Annuaire français de droit international, 51 : 433-463.

Newell, Peter, Nicky Jenner et Lucie Baker. 2009. «Governing Clean Development: A Framework for Analysis», Development Policy Review, 27 : 717-739.

O'Donnell, Guillermo. 1973. Modernization and Bureaucratic-authoritarianism: Studies in South American Politics. Berkeley, Berkeley University Press.

Pestre, Dominique. 2011. «Développement durable : anatomie d'une notion», Natures Sciences Sociétés, 19, 1: 31-39.

Revillard, Anne. 2018. «Saisir les conséquences d'une politique à partir de ses ressortissants. 
La réception de l'action publique», Revue française de science politique, 68, 3 : 469-491.

Rist, Gilbert. 2007. Le développement. Histoire d'une croyance occidentale. Paris, Presses de Sciences Po.

Société financière internationale. 2007. «Guidance Note 1: Social and Environmental Assessment and Management Systems G22. 》 <https://www.ifc.org/wps/wcm/connect/ topics_ext_content/ifc_external_corporate_site/sustainability-at-ifc/policies-standards/ performance-standards/ps1>. Page consultée le 15 mai 2015.

Tsayem Demaze, Moise. 2013a. «Au nom de la lutte contre le changement climatique : le mécanisme pour un développement propre et ses travers», VertigO - la revue électronique en sciences de l'environnement, 13, 2. <http://journals.openedition.org/vertigo/14020>. Page consultée le 28 mars 2018.

Tsayem Demaze, Moise. 2013b. «Les retombées du "Mécanisme pour un Développement Propre" pour les pays en développement : une faible réception de technologie et un développement durable vague», Les Cahiers d'Outre-Mer, 262, $2:$ 247-276.

Van Putten, Maartje. 2008. Policing the Banks: Accountability Mechanisms For the Financial Sector. Montréal et Kingston, Londres et Ithaca, McGill-Queens University Press.

World Commission of Dam. 2000. Dam and development: a new-framework for decisionmaking. <https://www.internationalrivers.org/sites/default/files/attached-files/world_ commission_on_dams_final_report.pdf>. Page consultée le 15 mai 2015.

\section{Notes}

1 L'auteure remercie les évaluateurs pour leurs commentaires qui ont contribué à l'amélioration et à la qualité de l'article.

2 Déclaration émise par le conseil du Mécanisme du développement propre le 28 septembre 2017. Voir la déclaration à la page <https://unfccc.int/news/forum-calls-for-clarity-on-futureof-clean-development-mechanism-under-paris-agreement>. Page consultée le 10 février 2019.

3 United Nations, Standard CDM, project standard for activities. CDM-EB93-H04-STAN.

$4 \quad$ L'article se base sur une enquête réalisée dans le cadre d'un postdoctorat au CERIC (UMR DICE 7318 - CNRS/Aix-Marseille Université), entre 2014 et 2016, sur le projet «International Grievance Mechanisms and International Law \& Governance», financé par le Conseil européen de la recherche dans le cadre du Septième programme-cadre de l'Union européenne (FP/2007-2013)/ERC Starting Grant Agreement n³12514.

5 Pour plus d'informations sur le fonctionnement des mécanismes de plainte, voir Lacuisse, 2017.

6 Informations issues du site internet de CONAGUA, le Conseil national de l'eau au Panama. <http://www.conagua.gob.pa/>. Page consultée le 12 février 2019.

7 Pour davantage d'informations sur la plainte, voir le cas Pando-Monte Lirio Hydroelectric Power Project (PN-MICI001-2010) sur le site internet du mécanisme de plainte de la BID. <https://www.iadb.org/en/mici/home>. Page consultée le 20 février 2019.

8 Entretien anonyme réalisé le 23 janvier 2015, Chiriqui, Panama. 
9 Entretien avec un plaignant anonyme réalisé le 24 janvier 2015, Panama. Traduction libre de l'auteure.

10 II s'agit de la résolution AG-0522-2006, du 21 septembre 2006.

11 Voir le rapport de conformité du cas Pando-Monte Lirio Hydroelectric Power Project (PN-MICl001-2010) sur le site du mécanisme de plainte de la BID.

12 II s'agit de la Loi 30 publiée en juillet 2010.

13 Décret-loi 18 de 2013.

14 Informations provenant de l'ASEP, I'Autorité de régulation des services publics au Panama.

15 Informations provenant de la base de données du «Clean Development Mechanism ». <http://cdm.unfccc.int/Projects/projsearch.html>. Page consultée le 1er septembre 2018.

16 Entretien anonyme réalisé le 24 janvier 2015, Chiriqui, Panama. Traduction libre par l'auteure.

17 La BM disposait d'une politique opérationnelle en matière de protection des Peuples indigènes jusqu'en 2016, politique qui a été remplacée par la norme environnementale et sociale $n^{\circ} 7$, nommée «Peuples autochtones/communautés locales traditionnelles d'Afrique subsaharienne historiquement défavorisées ».

18 Voir les plaintes Panama: Land Administration Project (First Request and Second Request) sur le site du mécanisme de plainte de la Banque mondiale. Disponible sur <http://ewebapps. worldbank.org/apps/ip/Pages/ViewCase.aspx?Caseld=12>.

19 Dans la base de données du MDP, le projet Bonyic est noté «rejeté » dans la liste des projets soumis par le Panama, tandis que le projet Chan 75 n'apparaît pas/plus dans la liste des projets soumis au MDP pour des raisons qui peuvent être autres que celle de l'impact de la mobilisation. En ce qui concerne le projet Chan 75, il a été planifié au début des années 2000 avec l'entreprise états-unienne AES et la non-soumission du projet est aussi à lier au fait que les États-Unis sont sortis des accords du climat entre temps.

20 Information provenant du site internet de l'organisation non gouvernementale International Rivers, qui a mis en accès libre divers courriers envoyés au MDP concernant la contestation de la certification de projets hydroélectriques au Panama. <https://www.internationalrivers.org/ resources/acd-comments-on-changuinola-1-chan-75-large-hydro-project-panama-3157>. Page consultée le 7 décembre 2017.

21 Entretien anonyme réalisé le 6 juin 2016, Panama City. Traduction libre de l'auteure.

22 II s'agit de la banque hollandaise FMO et la banque allemande DEG.

23 Ces deux ONG font partie des acteurs qui se sont mobilisés contre la certification des projets Chan 75 et Bonyic. Lors de la réalisation du terrain de cette recherche, elles participaient à la mobilisation contre la certification propre de Barro Blanco.

24 World Bank, OP 4.37, Safety of Dams, septembre 1996.

25 Base de données du «Clean Development Mechanism», consultée le 1er septembre 2017.

26 Voir International Rivers, «Large-Scale Power Projects Undermine the CDM»,

le 21 novembre 2012. <https://www.internationalrivers.org/blogs/246/large-scale-powerprojects-undermine-the-cdm>. Page consultée le 8 décembre 2017.

27 Voir le texte de la pétition en ligne sur le site de Carbon Market Watch : <https:// carbonmarketwatch.org/wp/wp-content/uploads/2018/10/Civil-society-letter-on-theClean-Development-Mechanism.pdf $>$. 